\title{
Estudio, Diseño e Implementación de un Sistema de Control de Velocidad y Monitoreo de Catarata de Carga de un Molino de Cerámica Mediante Procesamiento de Sonido en la Fábrica Graiman de la Ciudad de Cuenca
}

Christian Eduardo Romero Haro ${ }^{1}$, Iván Marcelo Escandón Deidán²,*

\section{Resumen}

El presente proyecto consiste en el control y monitoreo de la catarata de carga de un molino de bolas, cuya finalidad es la de reducir el tiempo de molienda, optimizando el movimiento del cuerpo moledor al interior del molino, basándose en el sonido que estos cuerpos producen durante la molienda.

Palabras clave: Molino de Bolas, Espectrogramas, Adquisición de Audio, STFT, Sistema de Control Discreto, Filtros Digitales.

\section{Abstract}

This project consistes of the control and monitoring of the cataract loading of a bad mill, whose purpose is to reduce the milling time, optimizing body movement inside the mill grinder, based on the sound these bodies produce during milling.

Keywords: Ball Mill, Spectrograms, Audio Acquisition, STFT, Discrete Control System, Digital Filters.

\footnotetext{
${ }^{1}$ Estudiante de la Carrera de Ingeniería Electrónica, Universidad Politeénica Salesiana, sede Cuenca.

${ }^{2, *}$ Ingeniero Electrónico, Docente de la Carrera de Ingeniería Electrónica, Universidad Politécnica Salesiana, sede Cuenca, Estudiante de la Maestria en Control y Automatizacion Industrial, Universidad Politécnica Salesiana. Autor para correspondencia: iescandon@ups.edu.ec

Recibido: 27 - Enero - 2012; Aprobado tras revisión: 20 - Mayo - 2012

Forma sugerida de citación: Romero, C. y Escandón, I. (2012). "Estudio, Diseño e Implementación de un Sistema de Control de Velocidad y Monitoreo de Catarata de Carga de un Molino de Cerámica Mediante Procesamiento de Sonido en la Fábrica Graiman de la Ciudad de Cuenca". INGENIUS. N, (enero/junio). pp 21-28. ISSN: 1390-650X
} 


\section{Introducción}

En los sistemas industriales, una rama muy interesante es la del procesamiento de sonido, ya que en la actualidad en muchos de los procesos de la industria se requiere de una optimización.

En la molienda discontinua de arcillas para la producción de revestimientos cerámicos se puede aplicar el procesamiento de sonido. Este tipo de sistema se debe a que en el interior de un molino de bolas el ambiente es altamente destructivo y no se puede colocar ningún tipo de sensor para monitorear los movimientos del material al interior del molino.

Un molino de bolas es un cilindro que gira sobre su eje horizontal, causando con ello el movimiento de los materiales en su interior. El material empleado como cuerpo moledor son piedras negras de río, las cuales mediante procesos de destrucción y abrasión que se dan al instante en que el molino de bolas gira, se encargan de disminuir el grosor de las tierras arcillosas hasta que éstas lleguen a un grosor determinado.[1]

El molino cuenta con un variador de frecuencia que comanda al motor al instante de su arranque y durante el proceso mantiene al molino en una velocidad constante. El proyecto se basa en el procesamiento del sonido que producen los cuerpos moledores en el interior del molino de bolas y el objetivo es optimizar el movimiento de estos materiales al interior del molino para de esta manera lograr disminuir los tiempos de molienda de un molino de la fábrica GRAIMAN.

\section{Molino de bolas}

El molino de bolas (Figura 1) es una carcasa cilíndrica que gira sobre su propio eje. Esta envoltura está llena aproximadamente hasta la mitad de objetos duros (medios de molienda), resistentes a la abrasión y de preferencia más pesados que el mineral a romper.[2]

Los medios de molienda se clasifican en:

- Esferas metálicas

- Barras metálicas

- Mineral de mayor tamaño y dureza

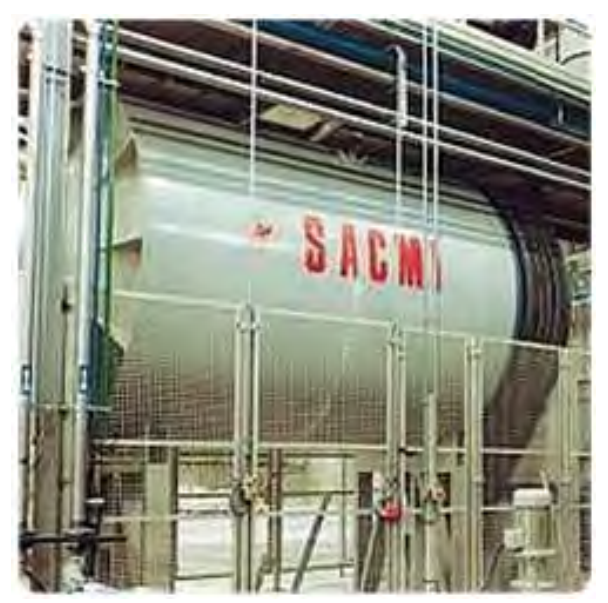

Figura 1. Molino de Bolas del fabricante Italiano SACMI.

El "medio de molienda", es aquel medio que permite la reducción del material. Es de mayor tamaño que el mineral a moler, pero de mucho menor que el tamaño del molino.

El mineral en su paso por el molino, puede ser fracturado mediante dos formas:

- Ciclo de cascada, el tipo de fractura es vía impacto preferencialmente.

- Cizalla (interior), el tipo de fractura es vía abrasión o astrición.

De esta manera los materiales menos resistentes se fracturan al interior del molino o se desgastan seguidamente hasta llegar al tamaño deseado, debido a los repetidos impactos y la abrasión a la cual están sometidos. 


\section{Procesamiento del sonido}

Un molino de bolas tiene cuerpos moledores de características similares (generalmente en forma y tamaño) en su interior. Una característica del sonido que producen los impactos del cuerpo moledor en la cara interior el molino es que se pueden escuchar estos sonidos. Esta característica es la que hace posible que se monitoreen los impactos que producen estos cuerpos en la cara interior del molino. Estos sonidos serán llamados los impactos de catarata. [1]

Para poder determinar al valor de frecuencia al que se dan los impactos se han utilizado algunos métodos. Uno de estos métodos es la FFT, pero esta no ha sido de gran ayuda, ya que lo que se requiere para determinar la catarata de carga del molino basándose en sonido es:

- Valores dentro de un ancho de banda de frecuencia.

- Tomar los valores en intervalos cortos de tiempo.

- Tener la capacidad de visualizar estos valores.

El problema de la FFT es que no da información de ningún tipo sobre el eje temporal ya que se hace sobre bloques de señal que pueden no contener información en un instante determinado y es de aquí de donde nace la idea de emplear espectrogramas.

Un espectrograma es una representación de una señal de acuerdo a las variaciones de la energía con respecto al tiempo y frecuencia. En el eje $\mathrm{X}$ va el tiempo, en el eje $\mathrm{Y}$ va la frecuencia, y en el eje $\mathrm{Z}$ va la amplitud o magnitud (véase Figura 2).

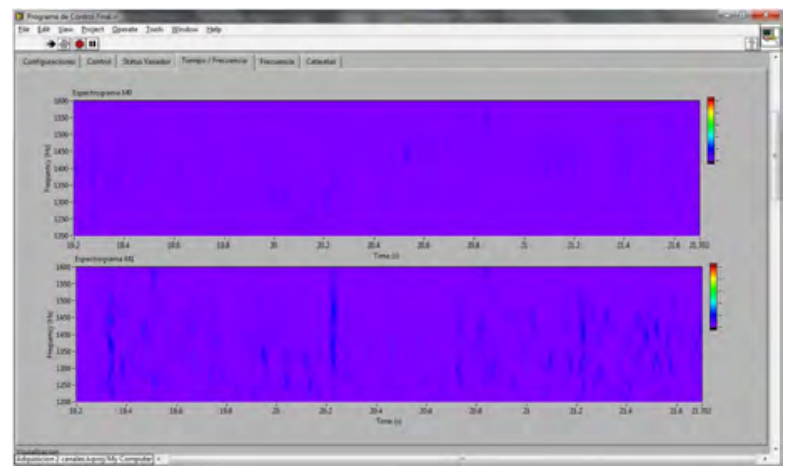

Figura 2. Espectrograma del sonido producido por el Molino de Bolas

Estos espectrogramas se utilizan a menudo en los sistemas de reconocimiento de voz, identificación de animales, evaluación de instrumentos musicales o sistemas de sonido y, en general, para el análisis de todo tipo de señales incluyendo el tratamiento de imágenes.

El método más común para la generación de espectrogramas es la STFT (Transformada de Fourier de Tiempo Reducido); es el método a emplear para el análisis espectral del proceso de molienda.

En la Transformada de Fourier de Tiempo Reducido la función a ser transformada es multiplicada por una función ventana que solo es diferente de cero por un pequeño período. La trasformada de Fourier (una función de una sola dimensión) de la señal resultante es tomada como una ventana que se desliza a lo largo del eje del tiempo, resultando en una representación de dos dimensiones de la señal. En el caso del tiempo discreto, la información a ser transformada podría ser dividida en pedazos o fragmentos, cada pedazo pasa por la transformada de Fourier, este resultado tiene valores complejos los cuales se colocan en una matriz que contiene la magnitud y fase para cada punto en tiempo y frecuencia. 
Esto puede ser expresado por la Ecuación 1.

$$
\operatorname{STFT}\{x[n]\}=\sum x[n] w[n-m] e^{-j \omega n}
$$

Donde, $x[n]$ es una señal y $w[n]$ es la ventana. De nuevo, el índice de tiempo discreto $m$ es normalmente considerado como un tiempo "lento" y usualmente no se expresa con tan alta resolución como con el tiempo $n$. La magnitud al cuadrado de la STFT origina el espectrograma de la función $x[n]$.

\section{Modelos del comportamiento de la carga al interior del moli- no}

\subsection{Modelo físico}

Este modelo pretende determinar la trayectoria del cuerpo moledor, considerando solo una bola y su punto final de caída. Se basa en un tiro parabólico como se muestra en la Figura 3 .

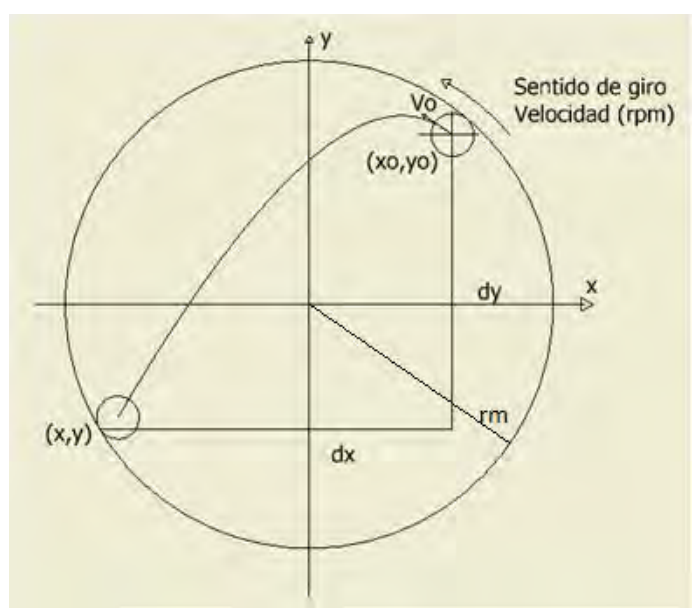

Figura 3. Gráfica que describe el modelo físico

Como se observa, se pretende determinar el trayecto del cuerpo moledor hasta que este impacta en la cara 3 interna del molino. Para ello debemos partir de las variables que consideraremos para nuestro análisis:
$V_{0}$ : velocidad del molino en $\mathrm{m} / \mathrm{s}$.

$\left(x_{0}, y_{0}\right)$ : Posición inicial del cuerpo moledor.

$d x$ : distancia total viajada en $x$.

$d y$ : distancia total viajada en $y$.

$(x, y)$ : coordenadas de impacto.

Las Ecuaciones que definen al modelo físico son la ecuaciones 2 y 3.

$$
x=-V_{0} \cdot \sin \theta \cdot t+r m \cdot \cos \theta
$$

$$
y=V_{0} \cdot \cos \theta \cdot t-\frac{1}{2} \cdot g \cdot t^{2}+r m \cdot \sin \theta
$$

\subsection{Modelo matemático}

Para la obtención del modelo matemático se emplearon algoritmos que permitan determinar la misma. Previo a la explicación del modelo se debe explicar el esquema de control que se va a manejar, el cual se muestra en la Figura 4.

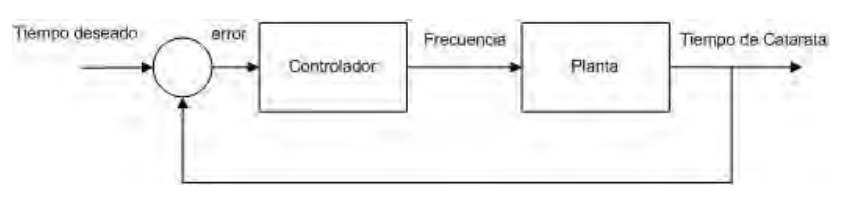

Figura 4. Esquema de control empleado

Donde la función a identificar es la de la planta. En la función de transferencia de la planta se incluye al software de procesamiento que encuentra los periodos de catarata, al molino y su variador de frecuencia. Estos subsistemas están ya incluidos en la planta.[3]

Para la identificación del sistema se empleará el toolbox de LabVIEW de Identificación de Sistemas, el cual facilitará nuestro trabajo. Se dará una breve descripción de la identificación de sistemas y los modelos planteados para la identificación. El modelo elegido es el Box-Jenkins (Figura 5), ya que ofrece 
una independencia entre el polinomio que involucra a la entrada con el error.[4]

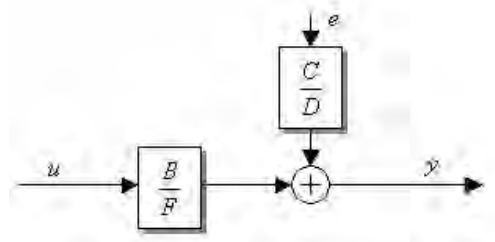

Figura 5. Diagrama de bloques del modelo Box-Jenkins

Al utilizar el algoritmo se ha obtenido la función de transferencia de la Ecuación 4

En base a la Ecuación 4, se procede a diseñar y simular el controlador.

\section{Experimentación y resultados}

\subsection{Condiciones habituales de funcionamiento}

Las condiciones bajo las cuales se realizaron las adquisiciones de sonido del sistema en condiciones habituales óptimas (las que se han venido manejando) son las siguientes:

- Carga del molino: siempre la misma.

- Vacío del molino: 1,3 m.

- Frecuencia de ingreso al motor de $60 \mathrm{~Hz}$ (12,5 RPM).

Los datos que se han medido son los que se presentan a continuación:

- Periodos de catarata (Segundos)

- Ángulos de impacto (Grados)

- Conteo de Impactos (Impactos/ min)

El comportamiento del periodo de catarata se presenta en la Figura 6

Como se observa en la Figura 6, la señal es demasiado ruidosa, por lo que se la ha sometido a un filtrado, que se observa por la señal en

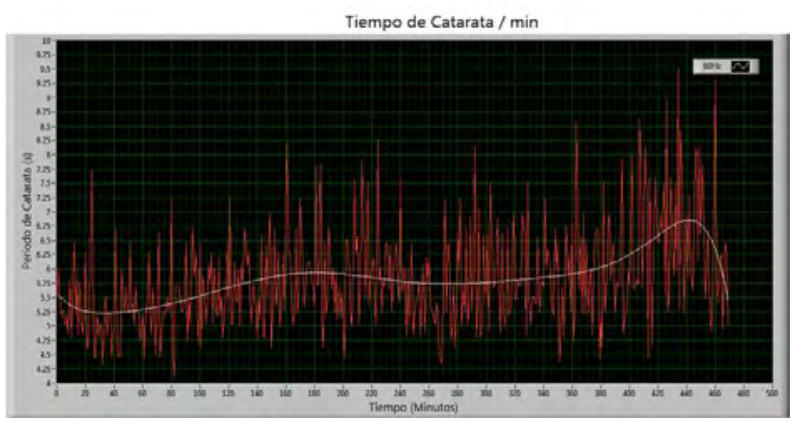

Figura 6. Periodo de catarata a velocidad de funcionamiento habitual

color blanco. La media del tiempo de catarata es de 5,73 segundos.

\subsection{Con el Controlador Implementado}

El rango de salida de variación de velocidad oscila entre 47 y $60 \mathrm{~Hz}(10,0$ y $12,5 \mathrm{RPM})$, en control automático se encarga de encontrar la salida de frecuencia para mejorar el movimiento de material al interior del molino. Las pruebas del controlador se han hecho basadas en las siguientes condiciones:

- Vacío del molino alto de 1,65 m (cercano al valor crítico).

- Carga especificada en párrafos anteriores.

Los resultados del ajuste de la variable a controlar (periodo de catarata) son los indicados en la Figura 7.

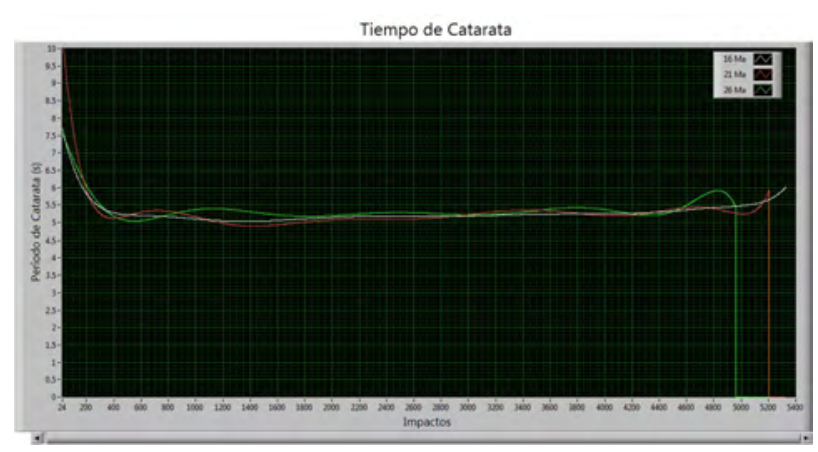

Figura 7. Periodo de Catarata con el sistema controlado

Con una simple inspección visual se puede observar una notable mejoría en el sistema, los 


$$
\begin{gathered}
y=\frac{0,046258 z^{-1}+0,0307 z^{-2}+0,03135 z^{-3}+0,026135 z^{-4}}{1+0,27712 z^{-1}+0,182268 z^{-2}+0,114069 z^{-3}+0,0742125 z^{-4}+0,0589077 z^{-5}} \cdot \mu(k)+\ldots \\
\ldots+\frac{1-0,11027 z^{-1}-0,07832 z^{-2}-0,042599 z^{-3}}{1+0,09712 z^{-1}+0,0698929 z^{-2}+0,0374809 z^{-3}+0,018426 z^{-4}} \cdot e(k)
\end{gathered}
$$

tiempos de catarata cumplen con el parámetro de error de estado estable, ya que la señal filtrada nos indica una estabilidad y mejora en el sistema. Además de una repetitividad de los resultados, indicando que el control se está realizando.

En la Tabla 1 se muestra los resúmenes de los datos presentados anteriormente en las Figuras 6 y 7 .

Analizando los datos de la Tabla 1 se obtiene varias observaciones:

- El parámetro que determina la finalización de los molienda es el residuo, actualmente debe estar entre un 9 y $10 \%$. Este valor depende directamente del tiempo de molienda, pero también depende de la cantidad de agua, es decir, si se coloca más agua de la requerido, el residuo disminuye mas rápido, pero se afectan los valores de densidad y residuo (tardan mas en llegar a sus valores establecidos).

- Las condiciones del clima afectan directamente al proceso. Por ejemplo, al estar húmedo y lluvioso el clima, se debe colocar menos agua en la fórmula, por lo que los mismos operadores deben cambiar la cantidad de agua necesaria.

- Con el sistema se logra que el material impacte una mayor cantidad de veces, lo que desencadenaria en menores tiempos de molienda.

- En cuanto al control, este optimiza el movimiento de los materiales al interior del molino, esto se puede notar claramente en las figuras. Los tiempos medidos e impactos contados nos muestran una notable mejoría del proceso, a pesar de no ser las condiciones óptimas de funcionamiento se ha logrado disminuir en al menos 1 segundo el periodo de catarata, que era lo esperado.

- Según los resultados de las pruebas se deben disminuir entre 30 minutos y una hora de molienda, lo cual cumple nuestro objetivo de disminuir el tiempo de molienda.

- Por factores de tiempo y disponibilidad en la fábrica no se han podido recopilar una mayor cantidad de datos de pruebas.

\section{Conclusiones}

Dentro de cualquier proceso es muy importante considerar todos los factores que influyen en él. Además de un control electrónico se deben considerar varios aspectos tales como: el personal que influye en el proceso y los factores climáticos, los cuales son de imperativa importancia si se trata de optimizar los procesos.

En el caso del proyecto realizado, no es la excepción, ya que se tiene una gran influencia de los factores que se involucran el proceso. Para tener un correcto funcionamiento del sistema se debe considerar factores climáticos y factores operativos. Del comportamiento del sistema bajo esas condiciones se puede concluir: 
Tabla 1. Resultados de las Pruebas de Control

\begin{tabular}{llll}
\hline Parámetro Medido & Prueba 1 & Prueba 2 & Prueba 3 \\
\hline Tiempo de Molienda & 8 horas & 8 horas & 8 horas \\
\hline Densidad & $160 \mathrm{gr} / \mathrm{cm}^{3}$ & $165 \mathrm{gr} / \mathrm{cm}^{3}$ & $164,3 \mathrm{gr} / \mathrm{cm}^{3}$ \\
\hline $\begin{array}{l}\text { Tiempo de estabilización de } \\
\text { viscosidad }\end{array}$ & $33 \mathrm{seg}$ & $52 \mathrm{seg}$ & $57 \mathrm{seg}$ \\
\hline Residuo & $7,3 \%$ & $8,5 \%$ & $7,8 \%$ \\
\hline Periodo de Catarata & $5,29 \mathrm{seg}$ & $5,3 \mathrm{seg}$ & $5,37 \mathrm{seg}$ \\
\hline Observación & $\begin{array}{l}200 \text { litros más de } \\
\text { agua (VM=1,65m) }\end{array}$ & $\begin{array}{l}300 \text { litros menos de } \\
\text { agua }(\mathrm{VM}=1,65 \mathrm{~m})\end{array}$ & $\mathrm{VM=1,75 \textrm {m }}$ \\
\hline
\end{tabular}

- Es imperativo mantener el molino de bolas en condiciones óptimas de funcionamiento, por ejemplo, al mantener un nivel adecuado de cuerpo moledor se tiene con qué moler las materias primas. Al instante de realizar el estudio se pudieron obtener curvas y comportamientos muy diferentes, medidos en distintas condiciones de cuerpo moledor. Al instante de realizar las pruebas de funcionamiento del sistema se obtuvo una optimización en el movimiento de material, que era lo dispuesto para el proyecto, pero al no tener el molino en condiciones optimas del nivel de cuerpo moledor, los tiempos que se manejan en la molienda tienden a subir y con ello la mejora que proporciona el proyecto tiende a mantener los tiempos en valores normales.

- La forma y uniformidad del cuerpo moledor tienen una gran influencia en el comportamiento de la catarata de carga del molino. Existe una gran diferencia entre cuerpo moledor empleado y el procesado (aluvid) donde se tiene un movimiento uniforme en la carga, además de tener una mayor superficie de contacto entre los cuerpos moledores. La uniformidad del movimiento se reduce notablemente al emplear las piedras de río, ya que no tienen una forma definida (unas pueden ser ovaladas, otras redondas y otras con puntas) y esto reduce la superficie de contacto además de afectar el volumen neto de cuerpo moledor ( $\sin$ considerar el vacio que hay entre las piedras)

- En etapas previas a la realización del proyecto se han realizado mediciones con un solo micrófono, al inicio colocándolo sin ningún tipo de aislamiento para el ruido, ya que en un ambiente industrial se tienen varias fuentes de ruido acústico. Por lo que se optó por colocar al micrófono en una "campana" de corcho, la cual favoreció al aislamiento de los ruidos externos. Una vez identificados los parámetros a medir, se vió la necesidad de cubrir un mayor sector del molino para poder identificar la posición de los impactos, por lo que se agregó un micrófono extra en una posición específica para poder cubrir mayor espacio del molino y detectar los impactos del cuerpo moledor.

- El tiempo de molienda depende directamente de la cantidad de agua que se cargue y de la humedad de las materias primas. De igual manera al instante de realizar las mediciones en una temporada de clima seco, donde la cantidad de agua 
a colocarse era la indicada para la formulación. Pero en las pruebas del sistema de control se realizaron en una temporada de lluvias donde la cantidad de agua cargada al molino se veía directamente influenciada por la humedad de las materias primas.

- Con la implementación del sistema de control se ha cumplido con el objetivo de reducir el tiempo de molienda, consecuencia de la optimización del movimiento de los materiales; esto trae resultados beneficiosos, ya que además de minimizar los tiempos de molienda hasta en una hora, se logra mantener el motor menos tiempo encendido en el mes (aproximadamente un $2 \%$ partiendo del consumo mensual), que brindó un ahorro energético.

En cuanto al modelado del sistema y su complejidad se ha recurrido a la utilización de métodos de identificación de sistemas dinámicos; estas herramientas han facilitado considerablemente el análisis y simulación del sistema. Como conclusión respecto a este tema sería importante el desarrollo de modelos matemáticos específicos para un molino de bolas.

El desarrollo del software ha sufrido varias modificaciones desde la primera vez que se realizaron pruebas, al no conocer bibliografía referente al tema se han obtenido varios comportamientos relevantes del sistema; y a su vez han sido usados en el sistema de detección de impactos para poder establecer la medición adecuada de las variables significativas.

\section{Referencias}

[1] J. Reyes, Cerámica Industrial, España. [Online]. Disponible en: http: //rua.ua.es/dspace/bitstream/10045/ 13558/1/CeramInd_JARLIQUA.pdf

[2] A. Olivares Mínguez, "Ingeniería avanzada para sistemas de control de ruido acústico mediante técnicas adaptativas," Tesis Doctoral, Universidad Politécnica de Madrid, 1998. [Online]. Disponible en: http://oa.upm.es/1184/1/ ANTONIO_MINGUEZ_OLIVARES.pdf

[3] I. Minchala. (2012, 23 de Marzo) Identificación, simulación, diseño e implementación de sistemas de control utilizando labview. [Online]. Disponible en: http://www.inele.ufro.cl/ apuntes/LabView/Manuales/Sistemas\% 20_Control \%20_Nivel.pdf

[4] n/d. (2012, 23 de Marzo) Sistemas automáticos de control. [Online]. Disponible en: http://www.juntadeandalucia. es/averroes/ies_sierra_magina/d tecnologia/bajable s/2 \%20bachillerato/ SISTEMAS \%20AUTOMATICOS \% 20DE \%20CONTROL.pdf

[5] A. Larco and P. Aguirre. (2012, 20 de Marzo) Introducción a la identificación de sistemas. Escuela Politécnica del Litoral. [Online]. Disponible en: http://www.dspace.espol. edu.ec/bitstream/123456789/11695/1/ Diapositivas \%20-Larco-Aguirre.pdf 